\title{
Cautionary Remarks on Using SPH to Model the ISM in Galaxies
}

\author{
P R Williams
}

Shanghai Astronomical Observatory, Chinese Academy of Sciences, 80 Nandan Road, Shanghai 200030, PR China

D K Churches, A H Nelson

Department of Physics and Astronomy, Cardiff University, Cardiff CF2 3YB, Wales, UK

\begin{abstract}
In many SPH simulations of galaxy formation a lower limit is imposed on the kernel radius $h$ equal to the gravitational softening length $\epsilon$. It has been found that such a constraint can in some circumstances compromise the spatial resolution to which hydrodynamical quantities are evaluated to the extent that the evolution of the gross features of a numerical galaxies are affected. Such effects can be avoided by allowing $h$ to evolve freely to maintain a roughly constant number of neighbours in SPH summations. Here we focus on how imposing a constraint on $h$ may affect the velocity field.
\end{abstract}

Figure 1 shows the gas mass fraction with $\nabla \cdot \mathbf{v}<0$, and the mean $\nabla \cdot \mathbf{v}$ for this mass versus time $t$ in a number of simulations using the constrained and unconstrained $h$ approaches. The initial conditions used were a sphere of uniform density given a Poisson spectrum of noise, in solid body rotation with a spin parameter of 0.06 . Initially $100 \%$ of the gas has negative $\nabla \cdot \mathbf{v}$ due to the collapse of the protogalaxy, but after the formation of the galactic disc both expansion and compression are present. For the unconstrained $h$ simulations roughly half the gas is in convergent flows; while there is a trend for increased mass of gas to be in convergent flows with increasing constraint on $h$. The frames on the left show that a large fraction of this gas resides in the central regions of the constrained $h$ galaxy simulations. Secondly, these data show how velocity gradients are systematically diminished with increases in the constraint on $h$; while the mean values are broadly consistent between the four unconstrained $h$ simulations shown.

These trends occur because when $h$ is not allowed to decrease naturally in high density regions, the SPH summations sample over a large range in velocities, especially in the central regions of the numerical galaxy disks. As a consequence, all quantities evaluated with SPH summations are smoothed. Due to the large values of $h$, shear viscosity becomes effective in transporting angular momentum radially outwards and gas mass inwards. Thus it is preferable that no constraint is applied to $h$; rather that $h$ is allowed to evolve freely such that SPH summations contain a roughly constant number of neighbours. 

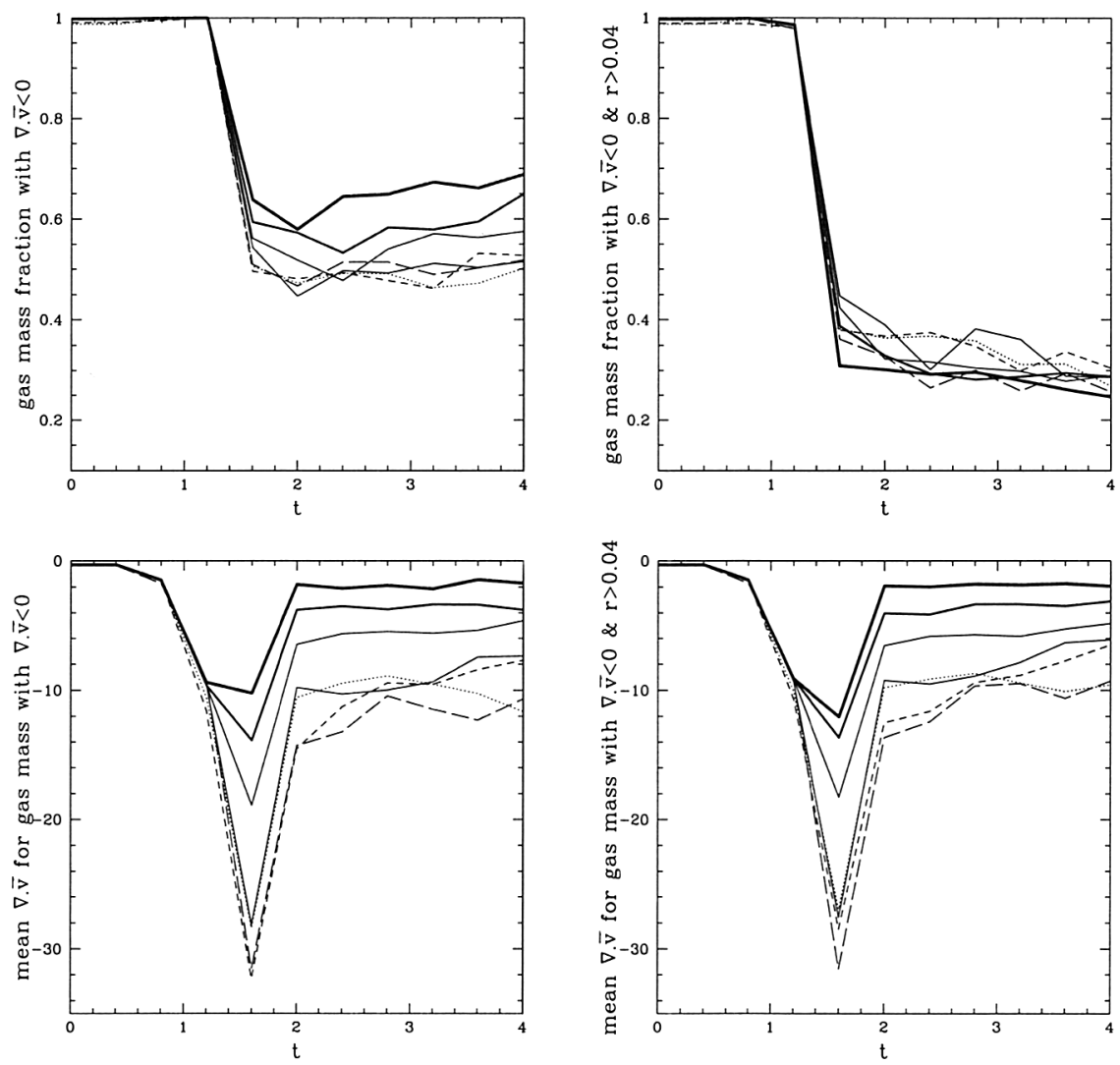

Figure 1. The gas mass fraction with $\nabla \cdot \mathbf{v}<0$, and the mean $\nabla \cdot \mathbf{v}$ for this mass versus time $t$. The right hand frames show these data excluding the central $4 \mathrm{kpc}$ of the numerical galaxies. Simulations which used constraints on $h$ of $h \geq \epsilon / 2, h \geq \epsilon$, and $h \geq 2 \epsilon$ respectively are shown with increasing line thickness. Simulations which use the unconstrained $h$ method with gravitational softening lengths of $1.5 \mathrm{kpc}$ and $1.0 \mathrm{kpc}$ are shown with a thin-solid and thin-dotted lines respectively (using 12000 particles in total). Simulations in which the total number of particles used is 12000,24000 , and 48000 , with a gravitational softening length of $1.0 \mathrm{kpc}$ are shown with thin-solid, dashed, and long-dashed lines respectively. 